\title{
A Statistical Analysis on the CEE (China) Grade Difference between Urban and Rural Candidates
}

\author{
Wu Cinan* \\ Science college of Guizhou university, Guiyang, China \\ *Corresponding author: cnwu@gzu.edu.cn
}

Received July 27, 2013; Revised August 04, 2013; Accepted August 07, 2013

\begin{abstract}
This paper, based on the Guizhou's data of recent years, is developed to reveal the difference in competitiveness between urban and rural candidates in the College Entrance Examination (CEE) of China. And Present the difference between urban and rural areas in different CEE subjects .After a complete analysis, which is based on solid data of CEE results, on the distributions and standard deviations of single-subject as well as total grades performed by each candidate category in both Arts and Science, and the correlation coefficients between each subject and the total grades, this paper concludes: Group differences exist in the overall performance; Outstanding differences exist in the grade constructions among different candidate groups, and the urban-rural contrast stands out in specific subjects. This paper also analyzes the corresponding relation of other statistical parameters concerning the CEE grades of both urban and rural candidates, and the relation between regional differences and CEE competitiveness. This paper also shows that if the direction of CEE reform is not taken properly, the candidate groups at a disadvantage in the current CEE system would probably be put in a worse position.
\end{abstract}

Keywords: fair educational opportunity, average grades, candidate groups, standard deviation, correlation coefficient

Cite This Article: Wu Cinan, "A Statistical Analysis on the CEE (China) Grade Difference between Urban and Rural Candidates." American Journal of Educational Research 1, no. 7 (2013): 240-246. doi: 10.12691/education-1-7-4.

\section{Introduction}

We are to study the difference of performance in all the subjects in CEE between Chinese urban and rural candidates, and a key issue is that if this difference varies from one subject to another or it stays steady in all the subjects. The conclusion cannot be drawn directly from the comparison between only one urban candidate group and one rural group. But if an overall statistical analysis is conducted upon a candidate group with a very large sample size, a certain conclusion can be made. The outcome can also lead to discussions about examination subject configuration and educational opportunity fairness. Fair educational opportunity is currently a hotly-debated issue in China. However, the increase in the total educational opportunities, especially higher education opportunities in China, does not narrow the gaps in educational opportunity as people have expected [1]. Since the mid-1990s, the educational fairness has been mainly reflected as the equality to education, but gaps in educational opportunities and educational qualities have widened [2]. China's College Entrance Examination (CEE) is a very important exam for candidates to be enrolled for higher education in China, but there are obvious disparities in the access to education between urban and rural areas, between men and women, and among various ethnic groups [3], as manifested in the CEE grades. Since admission to Universities in China is based mainly on the CEE grades, the grades are always a primary factor to higher education. Therefore, to explore the differences and correlation between CEE grades (and the grades of each subject) and the performances of different groups of candidates in the examination, has important practical significance for the reform of the content and means of CEE.

According to the Model of Status Attainment proposed [4], factors determining personal educational acquisition or resulting in unequal educational opportunities can be divided into three categories: family background, institutional factors and the length of education an individual receives [5]. Among existing empirical studies of the relationship between equality of educational opportunity and educational stratification, Samuel R. Lucas, after comparing the influences of family background on college enrollment of various age groups in the American society before and after the 1980s, found that even in the same premise of education expansion, the change in the educational policy could also change the unequal impact of family background [6]. With the increase of the total amount of educational resources, the degree of equality for those receiving lower levels of education might improve, but the university or other elite educational institutions are either dominated or monopolized by the rich and the powerful [7]. Family background and social status have great influence on the opportunities of the access to higher education. Prior to 
1978, China's educational opportunities had developed from an extreme inequality toward equality, but after 1978, the degree of inequality in the distribution of educational opportunities gradually increased, and the influence of family background and institutional factors on education rose constantly. The college enrollment expansion in 1999 benefited far more to the upper social strata than the lower ones [8]. Particularly, the resource advantage of the management class is gradually transformed into the educational opportunities of the next generation [9]. Therefore, for the urban and rural candidates in an increasingly polarized social environment, related researches have revealed that current CEE provides so few opportunities for children from lower social class to quality higher education[10,11].

Currently, the CEE candidates are mainly grouped according to urban and rural identities and their year of graduation (fresh candidates are those graduate in the same year they sit the CEE for the first time, and former candidates are those who sit the CEE again for a higher score after retaking the high school courses). Candidates from urban and rural regions possess different cultural capital (family cultural capital and personal education background), which will be reflected through CEE grades. First the total CEE grades may vary. If candidates from different groups have gotten the same total grades, their subject grades can show a different order.

The research curiosity lies in whether candidate group identity can result in apparent differences in subject test scores, and whether there are any other parameters in grade statistics correlating with the identity of the candidates.

So the following hypothesis is proposed in this study: after excluding intellectual factors, the family environment is the primary factor contributing to the differences between urban and rural CEE candidates, and plays a decisive role in the CEE grades. This paper is to make a comparison on the general performance in CEE (their average grades achieved) and other statistical
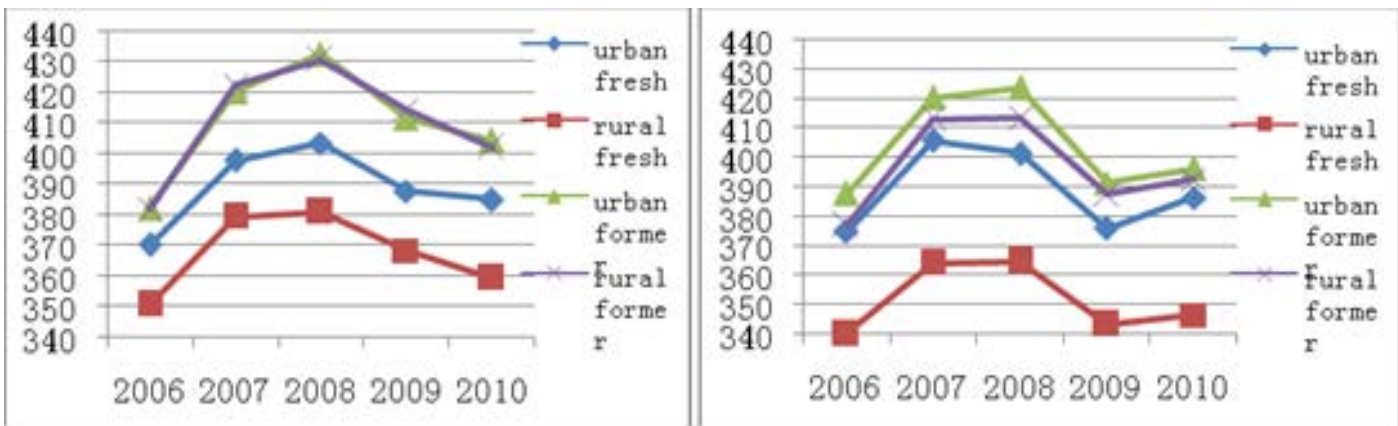

Figure 1. Difference in Total CEE Grades (left: arts candidates; right: science candidates) (2006 2010)

As can be seen from Figure 1, though there is fluctuation in average CEE total grades for both arts and science candidates during the five years due to the differences in question difficulty, it is very clear to see the consistent gap between their average total grades for those 4 groups.

For the fresh arts and science candidates, there is a fixed and large gap in grades between urban and rural candidate groups, but for the former candidates, regional gaps are small.

The figure shows that the average grades of former candidates are higher than fresh candidates, which means parameters [standard deviation (STDEV), and correlation coefficient (CORREL)] of arts and science CEE candidate groups according to current classification of region and year (urban fresh, rural fresh, urban former, and rural former), focusing on the gap between urban and rural candidates.

The yearly total number of candidates studied in this paper varies from 210,000 to 240,000. Among all the CEE candidates of a very large number, 99 percent have an age ranging between 18 years and 20 years old. The data is come from Guizhou, which is a province of China with a population of 39,000,000.

\section{Analysis of Average CEE Grades of Various Candidate Groups}

Located in the mountainous region of Western China, the urban-rural dual structure is rather obvious in Guizhou. There is a clear gap between urban and rural areas in the stage of compulsory education and senior secondary education, and typical differences exist in urban-rural gap for CEE candidate groups (urban fresh, rural fresh, urban former and rural former candidates).

The CEE grades from 2006 to 2010 in Guizhou Province have been used to work out the average grades of each subject of each group of candidates, with which this paper attempts to reveal the differences in the distribution of CEE grades of different candidate groups.

Guizhou Province follows the national common practice in designing CEE subjects and their grades, namely the following 4 subjects with a total CEE grade of 750 points altogether: Chinese (150 points), mathematics (150 points), English (150 points), and comprehensive ability (300 points for arts and science candidates respectively). Arts comprehensive ability (Arts c.a.) consists of politics, history and geography; Science comprehensive ability (Science c.a.) consists of physics, chemistry and biology. that after retaking the high school courses the former candidates could take full advantage of the educational resources and improve their CEE grades, narrowing their gap with urban former candidates.

Among CEE subjects, Chinese is relatively easier to score, so the gap in Chinese grades between urban and rural candidates (both arts and science) is generally not much (Figure 2). However rural fresh candidates still lag quite behind the three other groups, and this gap is significantly wider than the gap between urban fresh candidate group and former candidate groups. 

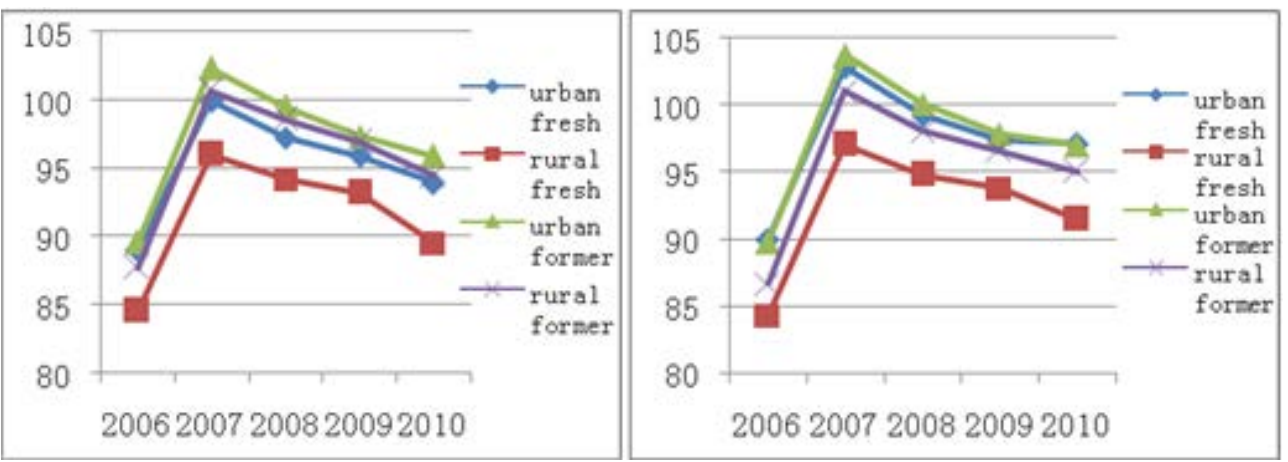

Figure 2. Difference in CEE Chinese Grades (left: arts candidates; right: science candidates) (2006 2010)
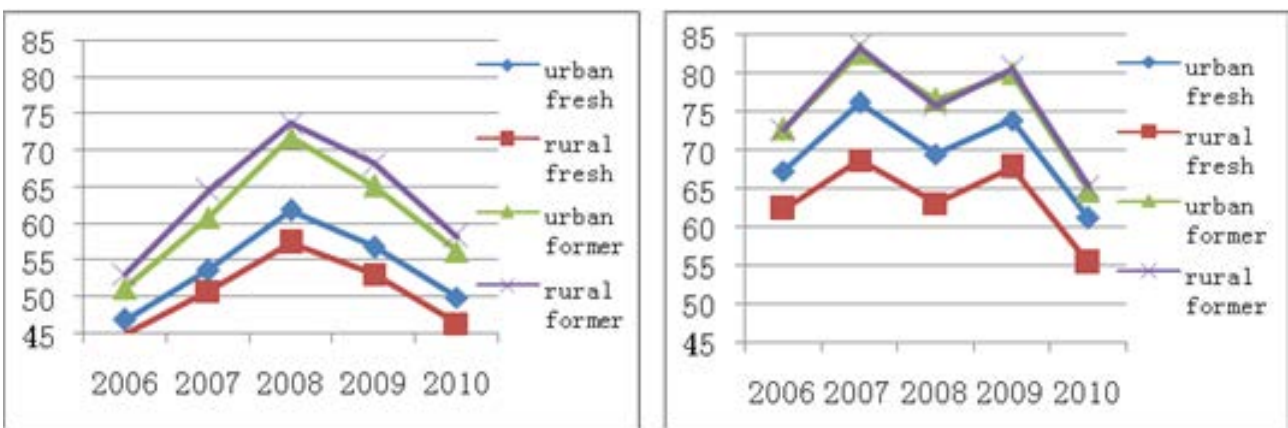

Figure 3. Difference in CEE Mathematics Grades (left: arts candidates; right: science candidates) (2006 2010)
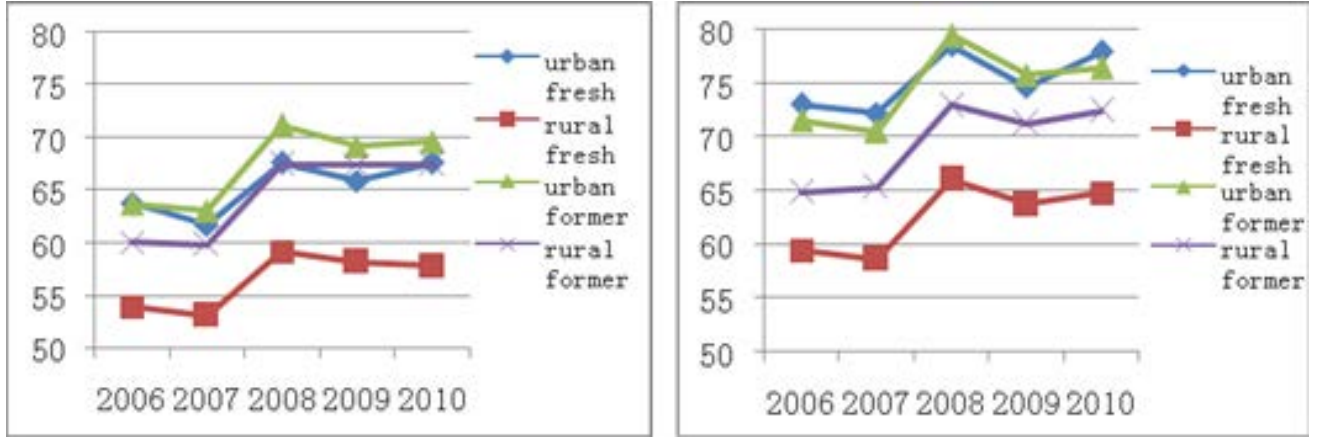

Figure 4. Difference in CEE English Grades (left: arts candidates; right: science candidates) (2006 2010)

As can be seen from Figure 3, there is a clear distribution order of mathematics grades for all the candidate groups, with the former candidates scoring much higher than the fresh ones. The gap between urban and rural areas exists stubbornly, but is much more moderate than the gap between former and fresh candidates. Urban fresh candidates do obviously better in mathematics than the rural fresh though, a glance at the grades of former candidates can reveal that the rural former arts candidates score slightly higher than the urban former, and the rural former science candidates can almost keep neck and neck with their urban competitors. All this shows that as long as there is opportunity for the rural candidates, they can do as well as their urban counterparts in relatively abstract subjects like mathematics.

For both the arts and science candidates, average English grades are generally quite low. However, there is a big gap between urban and rural candidates, and English even poses as a relative frustration for the rural former, especially in the science category. (Figure 4).
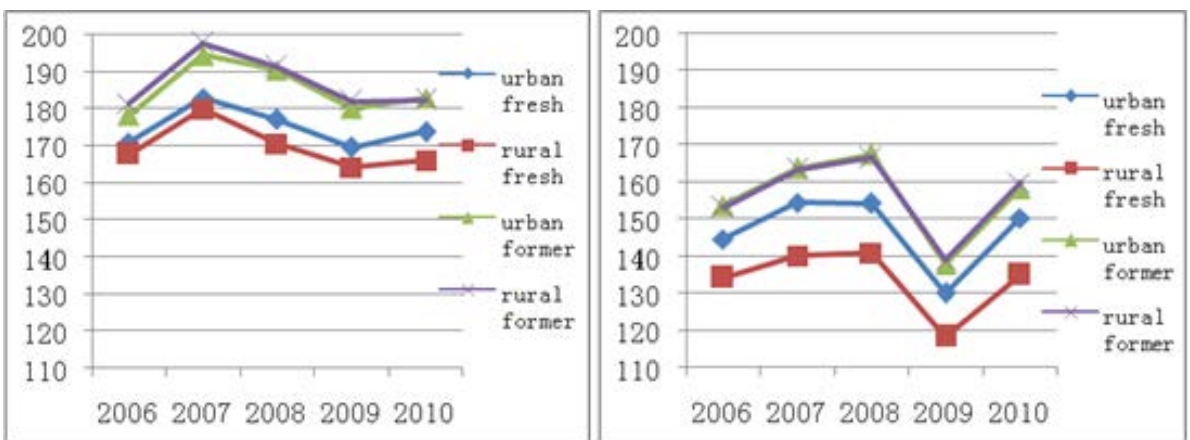

Figure 5. Difference in CEE Grades of Comprehensive Ability (left: arts candidates; right: science candidates) (2006 2010) 
In the science groups, there is little difference between the urban former and urban fresh, but an obvious gap between the rural former and rural fresh, which shows that urban candidates have a significantly higher English proficiency than rural candidates, and this gap cannot even be made up for through a retaking of high school courses.

As can be seen from Figure 5, the science candidates get obviously lower CEE grades in the subject of comprehensive ability. For the arts candidates, the urban fresh have scored higher than the rural fresh in the comprehensive ability test, but the gap between them, though small, shows no sign of narrowing.

For the science candidates, there is a constant and rather big gap between the urban fresh and rural fresh in this subject.

For the former candidates, be they arts or science, no obvious difference could be detected in the grades of the comprehensive ability test, which indicates that after retaking the courses the rural candidates could make a significant improvement in this subject. And this case also reveals that the rural candidates possess the same ability in learning this kind of intelligence-based subjects as their urban counterparts.

The above analysis of the CEE grades in the recent 5 years reveals that: (1) there is no disadvantage in intelligence or learning ability for the rural candidates, and the reason that they lag behind in the CEE grades is mainly because of the gap in educational resources and learning environment; (2) the disadvantages in the English subject of rural candidates cannot even be overcome through retaking the courses; (3) even though relative urban-rural differences exist in the Chinese subject, the gaps between the grades of urban and rural candidates are not very significance due to a relatively concentrated distribution of the scores; (4) except English, rural candidates can change their disadvantage in any other subjects through retaking the courses.

\section{An Analysis of the Standard Deviation of Grades of Various Candidate Groups}

The average grades reflect the performance of each candidate group in the CEE, and the average grade can be taken as: the expected mathematical value of some candidate randomly selected in a group without knowing any of his or her specific information; and its corresponding standard deviation shows how discrete the grades of one exam is scattered for a group.

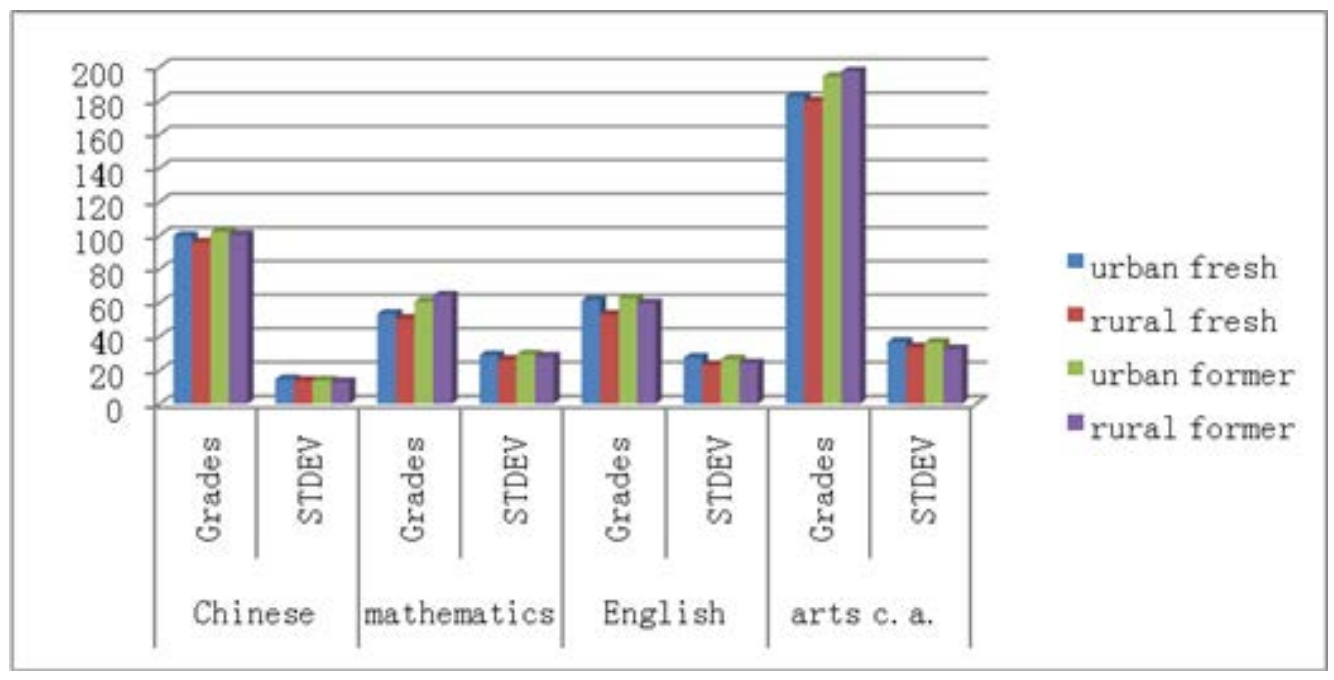

Figure 6. Overall average grades and standard deviation of each subject for arts candidates (2007)

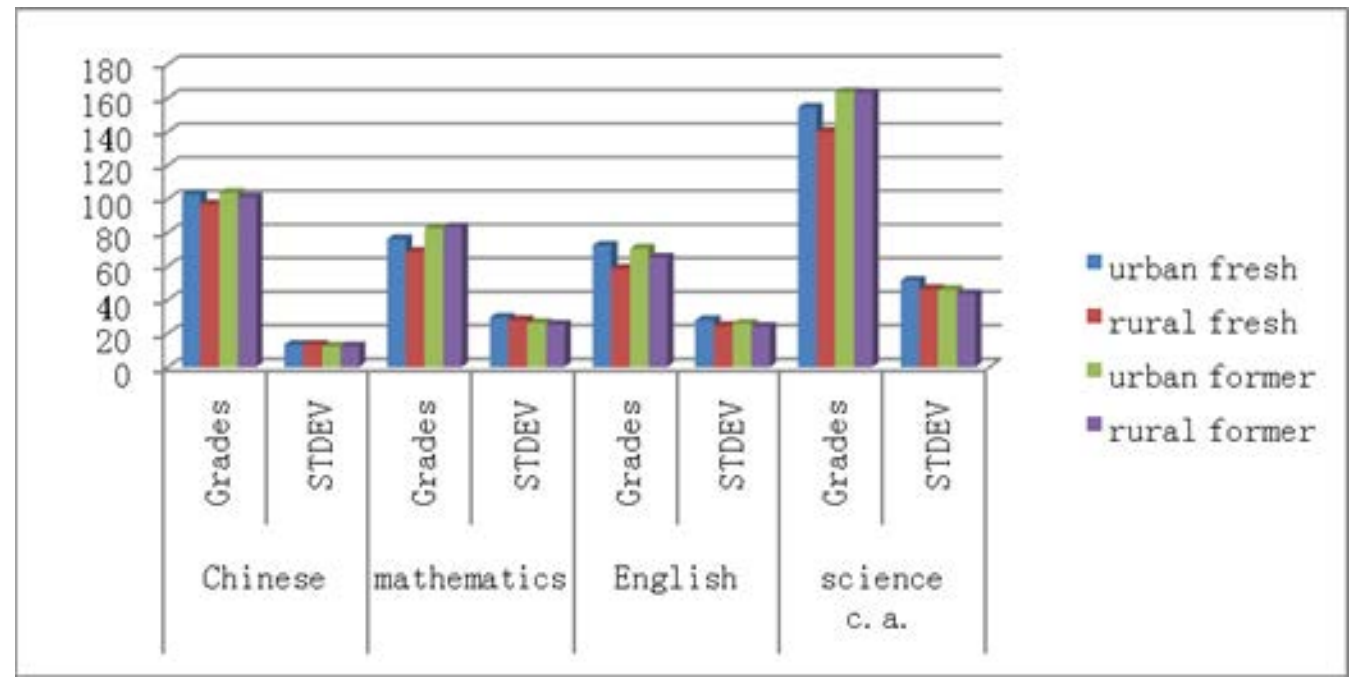

Figure 7. Overall average grades and standard deviation of each subject for science candidates (2007) 
As can be seen from Figure 6, with a comparison of the average grades of the candidates and the standard deviation of the grade distribution, obvious gaps can be found existing between the English, mathematics, and arts comprehensive ability grades respectively of various candidate groups. The highest Chinese graders are firstly the urban former candidates, then the urban fresh, rural former, and rural fresh the lowest; the sequence of average English grades ranks the same as the Chinese grade. For the mathematics and arts comprehensive ability grades, the rural former candidates have exceeded all the other groups at the top of the list, while the rest keep the same order as in Chinese and English grades. Hence, it is clear that the rural fresh candidates have scored the lowest in every subject in terms of overall average grades, and are the most disadvantaged group. However, after retaking the high school courses, those former candidates could score obviously higher, and the most prominent is the mathematics of rural former candidates. A comparison of the standard deviation of each subject of various groups of candidates shows the smallest difference in the Chinese grades, ranging between 13 and 15, while the standard deviation of mathematics, English and arts comprehensive ability grades, lying respectively at 26-30, 23-28, and 3337 , are clearly much larger than that of the Chinese grades.

Then the overall average grades and standard deviation of each subject for science candidates in 2007 is shown (Figure 7). After a comparison of the average grades and standard deviation, it is easy to see that there is a significant gap between English grades of various candidate groups.

The highest English graders are firstly the urban fresh candidates, then the urban former, rural former, and rural fresh the lowest; however, for distribution of the mathematics and science comprehensive ability grades, the urban-rural regional gaps of former candidate groups are small while the yearly gaps between former and fresh candidate groups are large.

The sequence of highest mathematics graders are rural former, urban former, urban fresh, and rural fresh; for science comprehensive ability, the highest is urban former, then the rural former, urban fresh, and the rural fresh. The sequence of highest Chinese graders is firstly the urban former, then the urban fresh and rural former, with the rural fresh score the lowest of all. It can be seen that the rural fresh candidate are in a relative disadvantaged position in each single subject, but the former candidates (both rural and urban) could make improvement in some of the subjects after retaking the courses, for example, mathematics of rural former candidates and the science comprehensive ability of urban former candidates. The most significant is that the rural former candidates score the highest in mathematics of all groups.

Among the standard deviation of the grades of each subject of various candidate groups, the deviation of the Chinese subject is the smallest at 12-13, greatly exceeded by English, mathematics and science comprehensive ability, which lie at 25-30, 24-28, and 43-51 respectively. The range of the deviation is also quite significant, and the science comprehensive ability subject has the most obvious deviation.

An analysis of the standard deviation of CEE grades in 2007 shows that: 1 , the Chinese grades of arts candidates have a relatively fixed order from urban fresh and former to rural former and at last rural fresh (sometimes the order can also be: urban former, rural former, urban fresh, and rural fresh); 2, a constant sequence appears in the English grades: from urban fresh, to urban former, then rural former and rural fresh; 3, there is also a constant order for the standard deviation of CEE grades of each subject and each group in all the years: urban fresh, urban former, rural former, and rural fresh.

\section{An Analysis of the Correlation Coefficient between the Subject Grades and Total Grade}

In test analysis the correlation coefficient, often a more oblivious statistical parameter, shows the consistency of the two sets of data. Therefore, the correlation coefficient between the subject grades and total grade of various candidate groups reflects the consistency between the subject grade and the total grade. In terms of the test subjects, the higher the subject's correlation coefficient is, the more dependent the total grade is on this subject; in terms of candidate groups, higher correlation coefficient indicates higher balance and less gap between grades of various subjects for this group.

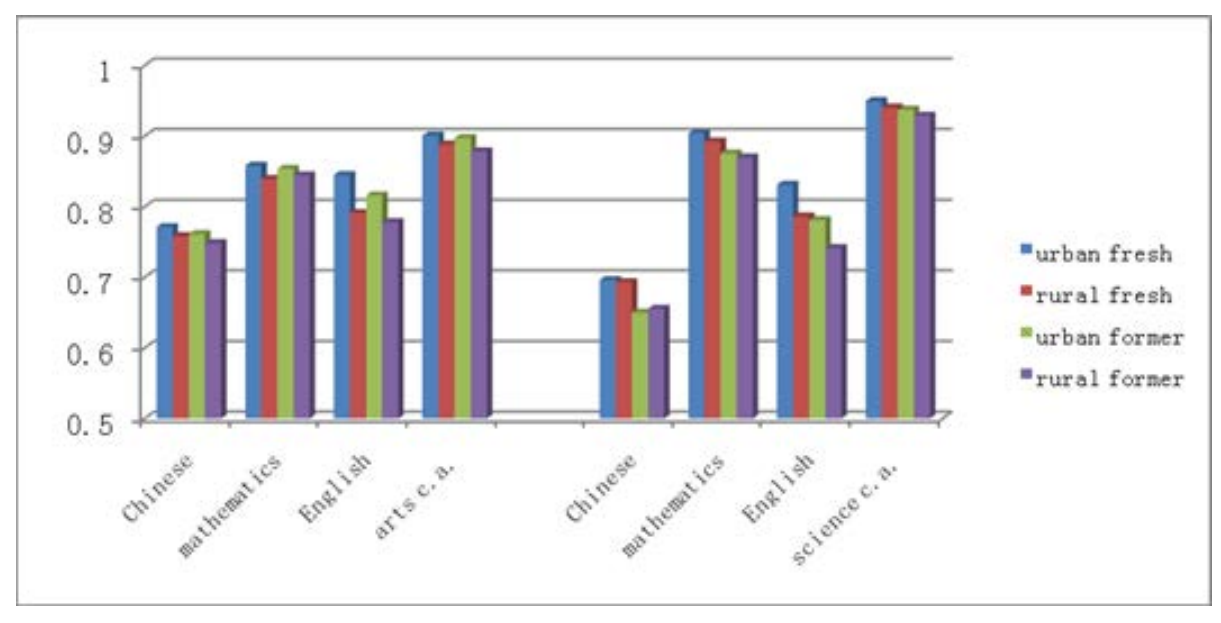

Figure 8. The correlation coefficient between the subject grades and total grade (2007)

Take the CEE grades in 2007 as an example, as can be seen from Figure 8, the distribution sequence of the subject grades' correlation coefficient (from low to high) with the total grades of the four arts candidate groups is: 
Chinese, English, mathematics, and arts comprehensive ability.

Among the arts candidate groups, the urban fresh candidates rank the highest of their correlation coefficient between all their subject grades and their total grades, and urban candidates have higher total grades correlation coefficient in any single subject than rural candidates. However, a comparison of the correlation coefficient of the English grades reveals a significant regional difference: the urban fresh candidates have a far higher correlation coefficient than the rural candidate groups.

For science candidate groups in 2007, the correlation coefficient between any single subject grades and total grades presents the following rising sequence: from Chinese, English, mathematics, to science comprehensive ability.

When comparing their correlation coefficient of the English subject, it is easy to find that the urban fresh are the highest. Their correlation coefficient of the Chinese subject shows a significant yearly gap between the fresh candidates and former candidates. However, only very small gaps exist in the correlation coefficient of science comprehensive ability for all the four groups.

Figure 8 also shows that for both the arts and science candidate groups in 2007, the comprehensive ability subjects are the highest in their single subject correlation coefficient, followed by mathematics and English, and Chinese ranks the lowest of all.

The comparison of the correlation coefficient between the single subject grades and total CEE grades of all candidate groups in the past years shows that the urban fresh ranks the highest because this group, of all the groups, are the most probable to keep balance between all testing subjects.

Poor studying environment and learning conditions of the rural candidates have made them relatively unable to improve their lame subjects as the urban candidates, but the priority of the total CEE grade in the enrolment would be less favorable to those with lame subjects, who, when rejected, become the former candidates if they want more try. As a result, the percentage of candidates with lame subjects is much higher in former candidate groups than in the fresh groups.

\section{Conclusion}

With the analysis above, a conclusion on the CEE subjects can be drawn as follows:

(1) The Chinese subject is the subject that shows the least difference between all the candidate groups with the test grades. Literature and writing, which generally reflect the talent of the students and the influence of students' temperament as well as family and school culture capital, should have been the most basic subject of CEE, but are not reflected in the CEE grades. It is known that Chinese is an urban-friendly subject (that is, a subject that more suitable to urban candidates rather than rural ones, note by the writer), the regional gap between urban and rural candidates remains very stable even though the concentrated distribution of the test scores cannot show much distinction. This subject is also experience-favored, because former candidates have slightly better performance over the fresh candidates, so the Chinese subject shows a feature of dependence on high cultural capital.

(2) The mathematical subject generally reflects the candidates' intelligence in the CEE, and is also a basis for their further study. However, because of the classification of arts and science categories, mathematics for science candidates is difficult and that for arts candidates is relatively simple. So it is hard to see which group it favors. Because of its experience- and training-favored characteristics, it does not show any malice to the working class typical of the exquisite symbols. Generally speaking, rural former candidates have performed the best in this subject so far.

(3) The average total English grades show a very discrete distribution in both arts and science groups with a large standard deviation and distinctive differentiation ability. At the same time, English is an urban-favored subject with great asymmetry between the urban and rural regions. The urban performance in English is significantly better than that of the rural candidates, and the fresh candidates also do better than the former. It is so far the most disadvantaged subject for the rural students, and this disadvantage cannot even be changed through retaking the course.

(4) Arts comprehensive ability has a relatively higher concentrated grade distribution and low differentiation. However, all the science candidate groups have scored relatively low in comprehensive ability, with a relatively dispersed grade distribution and high differentiation. Furthermore, this subject also has a larger correlation coefficient with the total grades. The comprehensive (art and science) ability subject is experience-favored, the highest graders are the rural former candidates, a subject with the most improvements for former candidates after more time and effort in the high school, and also the subject that the rural former candidates have done best.

\section{Discussion}

The above analyses reveal that, the difference of the performance between urban and rural candidates varies from one subject to another because of the influence of two kinds of educational environment and cultural capital. The biggest gap is found in the English subject between rural and urban candidates. But generally, rural candidates can, with more efforts in study, make up for the disadvantages of the weaker cultural capital caused by their family background. For instance, the gap is significantly reduced in the repeated candidate group. Through the study of this article, it can be clearly concluded that differences in family environment and educational background and inequality in the education process have been reflected in the CEE grades.

Regional difference between urban and rural areas is mainly reflected in the fresh candidates. The rural former candidates can even exceed their urban counterparts in some subjects, such as mathematics, which means that generally the rural students are not inferior to the urban students in terms of their intelligence and learning ability. Because of the complex structure of the former candidate group (after taking the CEE, a lot of urban candidates who have failed to be enrolled by normal universities or colleges would choose to study in some independent 
undergraduate colleges: the third batch enrolling colleges, as a result the urban former comprise only a far smaller percentage in the former candidate pool than those from the countryside), the differences in the performance of the former candidates is used just as a corroboration in this study.

An analysis of the CEE grades of each subject in the past years and their standard deviation reveals a relatively stable sequence in Chinese grades (from high graders to low graders: urban fresh and former, rural former, and rural fresh, or sometimes: urban former, rural former, urban fresh, and rural fresh) and a constant sequence in English grades (from high graders to low graders: urban fresh, urban former, rural former and rural fresh). The standard deviation of the CEE grades of various groups in these years also shows a fixed sequence in size (from big to small): the urban fresh, the urban former, the rural former, and the rural fresh. The standard deviation of urban fresh candidates tops the list in all groups because fresh candidates cover a wider range with both excellent and mediocre students. But former candidates are comprised mainly with those who failed the previous CEE but did not give up, while the very low graders would often lose hope and give up the second chance, so in this group very low grades are less likely to appear in large number. Compared with the rural fresh candidates, though the urban fresh have more high graders than the rural fresh, they still show a tendency of concentrated high and low graders because more academically poor students from the countryside simply dropped out in middle school or gave up the CEE. Actually the standard deviation of each subject is an accurate reflection of the gaps in the competitiveness inside a certain group.

Because of the differences in candidate groups, some of the subjects pose an obviously high cultural capital requirement and exclusion to the rural candidates, and a candidate group sequence appears in the following 3 parameters: their average grades, the grade standard deviation, and the correlation coefficient between subject grades and total grades, which indicates that the group category the candidates belong to can turn into, influence and be passed to the personal educational capital of the candidates (test scores and subject performance). Therefore, the CEE reform (particularly the reform of the adjustment of the test subjects) must be careful carried out. If an improper direction is taken, it will add insult to injury to the rural candidates. The suggestion is that the subject configuration of CEE does not have to be changed or adjust right now, but the contents in Chinese and English should be revised accordingly, getting rid of the problems relating to urban backgrounds as thoroughly as possible. At the same time, to promote the equity in education, educational resources in rural schools should be strengthened, and the learning environment should be improved.

\section{Acknowledgements}

This paper is part of the research achievement of China's MOE-Planned Fund Program “A study on the performance and behavior of certain group in CEE-A case study of Guizhou’s data” (11YJA880114).

\section{Statement of Competing Interests}

The authors have no competing interests.

\section{References}

[1] Hao Dahai, "A Study of Educational Disparity in Urban China (1949-2003)”. Social Sciences in China, 28(6): Chinese 94-107. 2007.

[2] Yang Dongping. "From Equality of Right to Equality of Opportunity: the Slot of Educational Equity in New China”. Peking University Education Review, Chinese 3(2):2-15. 2006.

[3] Dao Fudong, "Equal Educational Opportunity-Ideal or Reality?”, Chinese Social Sciences Today, Chinese p. 14. July 2 ${ }^{\text {nd }}, 2009$.

[4] Peter Michael Blau, Otis Dudley Duncan, The American Occupational Structure. New York: Wiley, 1967.

[5] Li Chunling, "Socio-Political Changes and Inequality of Educational Opportunity”. Social Sciences in China, Chinese 24(3): 86-98. 2003.

[6] Samuel R. Lucas, "Selective Attrition in a Newly Hostile Regime:The Case of 1980 Sophomores”. Social Forces. 75(2): 511. Dec.1996.

[7] Li Chunling, "Social and Political Changes and Inequality in Educational Opportunities: On the Impact of Family Background and Institutional Factors on Educational Attainment (1940-2001)”. Social Sciences in China. 24(4): 62-81. 2003.

[8] Zhang Yi. "Family Background Affecting People s Access to Education and Social Stratum Status”. Journal of Graduate School of Chinese Academy of Social Sciences, Chinese 32(4):82-91. 2010.

[9] Li Yu, Lin Hong, "Institutional Change and the Generative Mechanisms of Educational Inequality: Educational Attainment for Urban Children in China (1966-2003)”. Social Sciences in China, Chinese 28(1):72-83. 2007.

[10] Liu Yunshan, et al., "Selection of Elites: Views from Social status, Geographical variation, and Capital Gaining-Case Study on Farmers' children Who Get Admitted into Peking University (1978-2005)". Research On Education Tsinghua University, Chinese 30(5): 47-60. 2009.

[11] Gou Renmin, "Research on the Right of Equality in the Field of Higher Education”. Doctoral Dissertation. Law Department of Jilin University, Chinese p.58. 2006. 